\title{
In-Office Needle Arthroscopy Can Evaluate Meniscus Tear Repair Healing as an Alternative to Magnetic Resonance Imaging
}

\author{
Alex C. DiBartola, M.D., M.P.H., Alan Rogers, M.D., Peter Kurzweil, M.D., \\ Michael V. Knopp, M.D., Ph.D., and David C. Flanigan, M.D.
}

\begin{abstract}
Purpose: To assess the healing of horizontal cleavage tears of the meniscus 1 year after surgical repair and to determine what modality is best to determine healing. Methods: Patients were prospectively followed for 12 months after surgical meniscus repair using a circumferential compression stitch. Inclusion criteria were preoperative magnetic resonance imaging (MRI) evidence of horizontal cleavage tear, age between 18 and 50 years, and no concomitant anterior cruciate ligament reconstruction. Patients were excluded if they were $>50$ years old, had a meniscus tear pattern other than horizontal cleavage tear, and underwent concomitant ligament reconstruction. MRIs were performed l-year postoperatively for evaluation of repair healing. Preoperative and postoperative MRIs of tears were evaluated blindly by a musculoskeletal radiologist. In-office needle arthroscopy was performed at 6 months post-operatively. Results: Eight patients were included and had surgery between March 2016 and November 2017. There were 4 medial and 4 lateral meniscus tears. No patients had recurrence of preoperative symptoms or evidence of retear. Six repairs evaluated by inoffice needle (at 5.9 months postsurgery) arthroscopy demonstrated complete healing. Seven of the 8 patients had grade III changes on preoperative MRI, and 1 patient had grade IIc changes. On postoperative MRI, 5 of 7 patients had grade III changes, 1 patient had IIc changes, and 1 had IIb changes. There was no significant difference in the proportion of patients with grade III changes preoperatively compared with postoperatively $(P=.57)$. One of 8 patients with preoperative MRIs demonstrated extrusion where no patients demonstrate postoperative MRI evidence of extrusion $(P=.47)$. Conclusions: Horizontal cleavage meniscal tears repaired with a circumferential compression stitch demonstrate healing on in-office needle arthroscopy 6 months after surgery. No evidence of incomplete or failed healing was found. MRI at 1 year after surgery demonstrated residual tear evidence for all patients. Level of Evidence: IV, therapeutic case series.
\end{abstract}

From the Department of Orthopaedics (A.C.D., D.C.F.), Department of Radiology, (A.R., M.V.K.), Wright Center of Innovation (M.V.K.), Department of Orthopaedics Sports Medicine (D.C.F.), and Cartilage Restoration Program (D.C.F.), Ohio State University Wexner Medical Center, Columbus, Ohio; and Memorial Orthopaedic Surgical Group, Long Beach, California (P.K.), U.S.A.

The authors report the following potential conflicts of interest or sources of funding: D.C.F. reports grants and other income from Smith $\theta$ Nephew, other income from DePuy Mitek, grants and other income from Zimmer Biomet, grants and other income from Vericel, other from $M T F$, other income from $K C R N$, other income from Hyalex, grants and other income from Cartiheal, grants and other income from Anika Therapeutics, grants and other income from Aesculap, and grants and other income from Moximed, outside the submitted work. Full ICMJE author disclosure forms are available for this article online, as supplementary material.

Received December 28, 2020; accepted August 11, 2021.

Address correspondence to David C. Flanigan, M.D., OSU Sports Medicine, 2835 Fred Taylor Dr., Columbus, OH 43202.E-mail: David.flanigan@osumc. edu

(C) 2021 THE AUTHORS. Published by Elsevier Inc. on behalf of the Arthroscopy Association of North America. This is an open access article under the CC BY-NC-ND license (http://creativecommons.org/licenses/by-nc-nd/4.0/). 2666-061X/202048

https://doi.org/10.1016/j.asmr.2021.08.003
$\mathbf{M}$ eniscus tears are among the most commonly encountered and treated orthopaedic injuries. They occur in 2 per 1,000 patients per year, and partial meniscectomy is the single most common orthopaedic procedure performed in the United States alone. ${ }^{1,2}$ While numerous tear patterns exist, horizontal cleavage tears (HCTs) may account for up to $32 \%$ of all meniscus tears, and due to their complexity and poor healing potential, they have often been treated with partial or subtotal meniscectomy. ${ }^{3,4}$ However, modern all-inside repair devices using circumferential compression sutures have demonstrated superior load to failure compared with other techniques, while limiting the morbidity of additional surgical exposure required for inside-out repair techniques. ${ }^{5,6}$

Meniscus healing after repair, regardless of the tear pattern or repair technique, remains difficult to assess, and there are a wide variety of methods used..$^{7-9}$ Clinical evaluation, patient symptomology, return to function and/or sport, and patient future surgeries for 
meniscus-related pathology have been used to assess meniscus repair success. ${ }^{8,10}$ Magnetic resonance imaging (MRI) and second-look arthroscopy remain the primary options to assess repair healing, ${ }^{11,12}$ although second-look arthroscopy remains the gold standard. ${ }^{13-15}$ MRI does allow for an easier, quicker, and noninvasive assessment compared with secondlook arthroscopy, which necessitates another procedure and is often not feasible or indicated in the asymptomatic patient for the evaluation of repair status. In addition, in-office needle arthroscopy (IONA) has become increasingly popular for the evaluation of cartilage injuries, ligamentous status, and meniscus pathology. ${ }^{16-20}$ The IONA procedure allows for the direct visualization and evaluation of intra-articular knee structures, including the status of the surgically repaired meniscus.

HCTs have historically been thought to have poor healing potential due to tear characteristics lack of adequate repair techniques. However, newer treatment strategies and devices are being employed. Nevertheless, there remains a paucity of data regarding the arthroscopic evaluation of healing after HCT repair. Furthermore, to our knowledge, there are no previous publications evaluating HCT repairs with IONA and comparing these results to an assessment of healing using MRI.

The primary purposes of this study were to assess the healing of HCTs of the meniscus 1 year after surgical repair and to determine what modality is best to determine healing. It was hypothesized that patients undergoing repair of HCTs using all-inside circumferential compression sutures would demonstrate healing on IONA 6 months after repair, and that these results with be similar to the assessment of healing at 12 months with MRI.

\section{Methods}

Patients who underwent surgery for HCTs between March 2016 and November 2017 by a single surgeon (D.C.F.) were identified. This study was approved by our institutional review board and included patients enrolled at a single institution. The inclusion criteria were having a preoperative MRI evidence of HCTs, aged between 18 and 50 years old, and had no concomitant anterior cruciate ligament reconstruction performed at time of surgery. Patients were excluded if they were $>50$ years in age, had a meniscus tear pattern other than horizontal cleavage tearing, and underwent concomitant ligament reconstruction at time of surgery. All patients were followed for a minimum of 12 months.

\section{Surgical Technique}

All patients were evaluated clinically and with MRI before surgery. A single sports medicine fellowship-trained orthopaedic surgeon (D.C.F.) performed all procedures. A standard diagnostic arthroscopy was performed for all patients to evaluate for concomitant cartilage damage and other intraarticular injuries. An electric shaver and meniscus punch were used to trim areas of each patients' tear before repair. HCTs were repaired using a circumferential compression stitch (Ceterix NOVOSTITCH; Smith \& Nephew, Andover, MA) (Fig 1). No augmentation procedures to stimulate healing were performed.

All patients followed a standard and institutionspecific online published postoperative protocol for therapy after meniscus repair that began with a period of non-weight-bearing ( 4 weeks) followed by a period of partial weight-bearing ( 2 weeks) and a progressive return to full activity by 4 to 6 months. Clinical repair failure was defined as recurrence of symptoms necessitating revision surgery.

\section{Data}

Demographic data were recorded for the entire patient cohort. Patients with HCTs were enrolled and prospectively followed after surgical repair using a circumferential compression stitch (Ceterix NOVOSTITCH; Smith \& Nephew). There were no concomitant procedures performed at the time of arthroscopic repair other than chondroplasty in 1 patient. All patients had a preoperative MRI demonstrating an HCT. MRIs were performed at 12 months postoperatively for evaluation of repair failure or the extent of repair healing.

All preoperative and postoperative MRI studies were evaluated by a fellowship-trained musculoskeletal radiologist using a previously published MRI grading system (grades I-III, Table 1) as reported by Lotysch et al. ${ }^{21}$ MRIs were evaluated for abnormal signal intensity within the menisci indicative of meniscal tearing or healing. The radiologist was blinded as to the patient's demographic information and preoperative versus postoperative status. Patients underwent IONA (mi-eye; Trice Medical, Malvern, PA) to assess repair healing as a planned component of their 6-month postoperative visit. Healing on IONA was assessed independently and in a blinded fashion by 2 reviewers (one sports medicine fellowship-trained orthopaedic surgeon and 1 senior orthopaedic surgery resident) using criteria (completely healed, incompletely healed, failed healing) set forth by Morgan et al. ${ }^{22}$ and Kanto et al. $^{23}$ Specifically, healed repairs demonstrated no defects or hypermobility, incompletely healed repairs had partial defects and were stable, and failed repairs were mobile secondary to either failed repair site or secondary tears. ${ }^{22}$

\section{Statistical Analysis}

Descriptive statistics were generated for the entire sample and all statistical analysis and tests were performed using STATA, 14.0 (StataCorp, College Station, TX). A power analysis was not performed due to the 

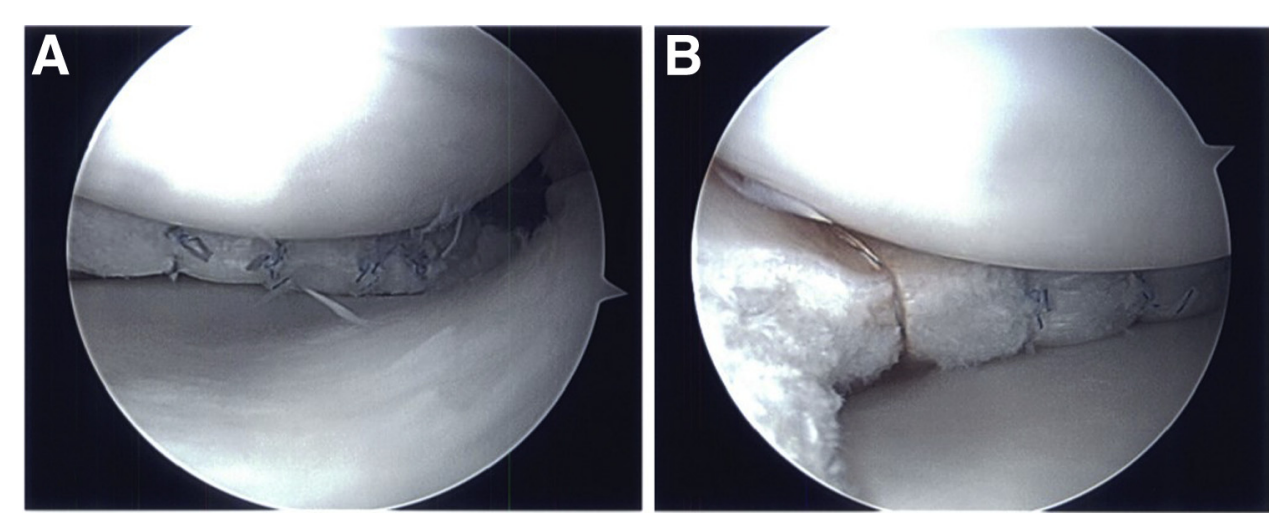

Fig 1. Posterior (A) and midsubstance (B) views, respectively, of a repaired meniscus horizontal cleavage tear, taken intraoperatively after repair.

unique and explorative nature of the study. Comparison of categorical variables preoperatively and postoperatively were performed via Fisher exact tests due to the low number of patients.

\section{Results}

Eight patients met inclusion criteria and were enrolled in the study. The mean age at the time of HCT repair was 32 years (standard deviation [SD] 9.2) (Table 2), with a mean follow-up of 27.7 months (SD 14.4). All but 1 patient presented with preoperative mechanical symptoms described as presence of locking, catching, and/or clicking. Mean body mass index was 25.4 (SD 3.0). Injury mechanism included wrestling $(\mathrm{n}=1)$, weightlifting $(n=1)$, jumping exercise $(n=1)$, soccer $(n=1)$, and unknown $(\mathrm{n}=4)$. One patient underwent a concomitant chondroplasty at the time of HCT repair surgery. Two patients had previous knee surgery on their operative knee $(\mathrm{n}=1$ unknown, $\mathrm{n}=1 \mathrm{ACL}$ reconstruction). There were 4 medial HCT repairs and 4 lateral HCT repairs. One patient developed a superficial wound infection that resolved after 10 days of oral antibiotics, Grade 1 (temporary disadvantage, no reoperation) according to Gosling and Gouma's ${ }^{24}$ classification system. No other complications were observed.

Table 1. MRI Evaluation of Meniscus Healing

\begin{tabular}{ll}
\hline Grade & \\
\hline 1 & $\begin{array}{l}\text { Small focal area of hyperintensity, no extension to the } \\
\text { articular surface } \\
\text { Linear areas of hyperintensity, no extension to the } \\
\text { articular surface }\end{array}$ \\
$2 \mathrm{a}$ & $\begin{array}{l}\text { Linear abnormal hyperintensity with no extension to the } \\
\text { articular surface }\end{array}$ \\
$2 \mathrm{~b}$ & Abnormal hyperintensity reaches the articular surface on \\
& a single image \\
$2 \mathrm{C}$ & $\begin{array}{l}\text { Globular wedge-shaped abnormal hyperintensity with no } \\
\text { extension to the articular surface }\end{array}$ \\
3 & Abnormal hyperintensity extends to at least one articular \\
& surface (superior or inferior) and is referred as a \\
& definite meniscal tear.
\end{tabular}

MRI, magnetic resonance imaging.
IONA (6 patients; 4 medial repairs, 2 lateral repairs) was completed (visualization only) at a mean of 5.9 months (SD 0.39 months) after the index surgery as planned per the study protocol. Tears were not probed. All 6 HCT repairs (100\%) evaluated by IONA demonstrated complete healing, which was significantly greater than tears that demonstrated no or partial healing $(0 \%)$, based on criteria published by Morgan et al. ${ }^{22}$ and Kanto et al. ${ }^{23}$ (Fig 2) (Fisher exact test; $P=$ .002). No repairs failed or demonstrated partially healing. At final follow-up, no patients had recurrence of these symptoms or clinical evidence of retear.

Postoperative MRIs in 7 patients were completed at a mean of 12.4 months (SD 0.88 months) per study protocol to evaluate for HCT repair healing using criteria set forth by Lotysch et al. ${ }^{21}$ On preoperative MRI, 7 of 8 patients demonstrated grade III meniscal signal changes and 1 patient demonstrated grade IIc signal changes (Table 3). Grade IIc abnormalities demonstrated on MRI as signal hyperintensity on water-weighted sequences without extension to the articular surface. These changes were accompanied by meniscal extrusion (as defined by greater than $3 \mathrm{~mm}$ of peripheral margin meniscal tissue extension beyond the external aspect of the tibia) this patient (Fig 3). On postoperative MRI evaluation, 5 of 7 patients demonstrated grade III signal changes, 1 patient demonstrated grade IIc changes, and 1 grade IIb changes. There was

Table 2. Demographical Data

\begin{tabular}{lc}
\hline $\begin{array}{l}\text { No. Subjects } \\
\text { Sex }\end{array}$ & 8 \\
$\quad$ Male & 5 \\
$\quad$ Female & 3 \\
Mean age at surgery, y (SD) & $32.1(9.2)$ \\
Mean BMI (SD) & $25.4(3)$ \\
Percent medial HCT repair & $50 \%(\mathrm{n}=4)$ \\
Percent lateral HCT repair & $50 \%(\mathrm{n}=4)$ \\
Percent smoker & $12.5 \%(\mathrm{n}=1)$ \\
Mean follow-up, mo (SD) & $27.8(14.4)$
\end{tabular}

BMI, body mass index; HCT, horizontal cleavage tear; SD, standard deviation. 

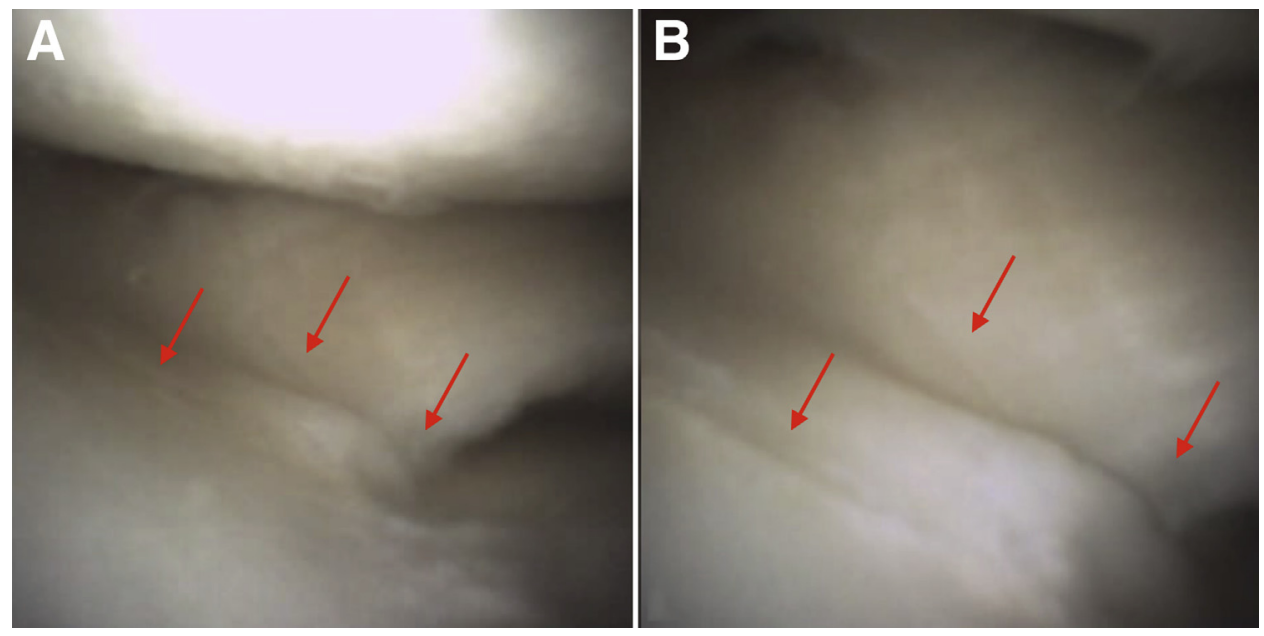

Fig 2. Full-perspective (A) and zoomed (B) views, respectively, of a healed right knee lateral meniscus horizontal cleavage tear repair in a 25 -year-old male patient using inoffice needle arthroscopy 5.6 months after tear repair. Red arrows indicate areas of healing about previous tear.

no significant difference in the proportion of patients with grade III changes preoperatively (7 of 8 patients) compared with postoperatively ( 5 of 7 patients) (Fisher exact test; $P=.57$ ). One of 8 preoperative MRI studies demonstrated evidence of meniscal extrusion whereas none of the postoperative MRI studies demonstrated meniscal extrusion (Fisher exact test; $P=.47$ ).

\section{Discussion}

This study suggests that HCTs treated with circumferential compression sutures heal with a low failure rate ( 0 failures at mean follow-up of 2.2 years) as evaluated by IONA at 6 months. In addition, IONA demonstrated valuable information in the evaluation of postrepair healing status.

Previous studies have evaluated different modalities to assess meniscus healing after surgical repair. ${ }^{7-9}$ These include second-look arthroscopy, IONA, MRI, MR arthrography (arthro-MRI), and CT arthrography (arthro-CT). ${ }^{7-9,25,26}$ However, these studies have demonstrated inconsistency in their ability to accurately and reliably assess the healing process. For instance, Almeida et al. ${ }^{27}$ evaluated meniscus repair healing after outside-in repair on 8 patients using arthro-MRI, arthro$\mathrm{CT}$, and MRI alone. They found that standard noncontrast MRI demonstrated healing in $50 \%$ of their patients as compared with a $75 \%$ rate of healing when using arthroCT or arthro-MRI. Other studies have also corroborated the notion that MRI is suboptimal for the evaluation of meniscus healing, demonstrating similar healing rates when using routine noncontrast MRI. ${ }^{10,28,29}$ Second-look arthroscopy remains the gold standard.

Interestingly, the repair of HCT has demonstrated good outcomes despite the inconsistent ability to demonstrate a healing response on MRI. ${ }^{28,30}$ It may be speculated that fibrous tissue associated with the healing process may still appear as increased signal intensity on MRI, although the present study is not designed to assess this.
Numerous studies evaluating second-look arthroscopy have demonstrated that this modality yields important and accurate information regarding meniscus healing status. ${ }^{8,23,31}$ Tachibana et al. ${ }^{32}$ evaluated 62 longitudinal or double longitudinal meniscus tears for healing using second-look arthroscopy and documented a 74\% complete healing rate and a $15 \%$ partial healing rate. Ahn et al. ${ }^{33}$ evaluated healing of 11 patients' (original cohort $\mathrm{n}=34$ ) HCT repairs (using vertical sutures with FASTFIX anchors (Smith \& Nephew Endoscopy, Andover, MA) with second-look arthroscopy at a mean of 45.6 months after repair and found complete healing in $73 \%$ of patients, partial healing in $18 \%$ of patients, and healing failure in $9 \%$ of patients. However, second-look arthroscopy is not without drawbacks, including increased costs from surgery and the usual risks associated with anesthesia and arthroscopic procedures. Data exit in the literature to support the use IONA for diagnostic purposes in knees and procedures such as partial meniscectomies. ${ }^{16,17,19,34}$ However, there is a lack of information regarding the evaluation of healing after HCT repair. The present study demonstrated a $100 \%$ healing rate after repair using a circumferential compression suture all-inside device.

At 6 months postoperatively, the IONA documented excellent healing in all 6 patients with HCTs who

Table 3. Preoperative and Postoperative MRI Grade

\begin{tabular}{lccc}
\hline $\begin{array}{c}\text { Mean Outcome } \\
\text { Scores }\end{array}$ & $\begin{array}{c}\text { Preoperative } \\
\text { Grade }\end{array}$ & $\begin{array}{c}\text { Postoperative } \\
\text { Grade }\end{array}$ & Pre-/Postextrusion \\
\hline Patient 1 & 3 & 3 & $\mathrm{~N} / \mathrm{N}$ \\
Patient 2 & 3 & $2 \mathrm{~b}$ & $\mathrm{~N} / \mathrm{N}$ \\
Patient 3 & 3 & 3 & $\mathrm{~N} / \mathrm{N}$ \\
Patient 4 & 3 & 3 & $\mathrm{~N} / \mathrm{N}$ \\
Patient 5 & $2 \mathrm{C}$ & 3 & $\mathrm{Y} / \mathrm{N}$ \\
Patient 6 & 3 & $2 \mathrm{C}$ & $\mathrm{N} / \mathrm{N}$ \\
Patient 7 & 3 & 3 & $\mathrm{~N} / \mathrm{N}$ \\
\hline
\end{tabular}

$\mathrm{N}$, no; MRI, magnetic resonance imaging; Y, yes. 
Fig 3. Sagittal intermediateweighted fat-saturated (A) and coronal intermediate-weighted fatsaturated (B) magnetic resonance images of a 33-year-old female patient with a lateral meniscus horizontal cleavage tear before repair. This abnormality is consistent with grade 2c changes, globular wedgeshaped abnormal hyperintensity with no extension to the articular surface, with extrusion. Red arrows indicate tear region.


underwent the procedure. Ogawa et al. ${ }^{30}$ demonstrated poor healing as seen on MRI after HCT repair, which is in line with the present study's MRI data demonstrating a poor healing response on MRI at 12 months postoperatively. However, Ogawa et al. also demonstrated excellent clinical outcomes using Lysholm and Knee Injury and Osteoarthritis Outcome Scores at a mean follow-up of 35 months, ${ }^{30,35}$ and while the evaluation of specific clinical outcomes scores was beyond the scope of this study, our patients were noted to be symptom-free at a mean follow-up of 27.8 months postoperatively with no clinical failures. Thus, the clinical success of this study is in-line with previous published data and supports the repair of HCTs, regardless of postoperative MRI findings. It should also be noted that 1 patient in the current study did demonstrate meniscal extrusion preoperatively; nevertheless, no extrusion was demonstrated on postrepair MRI scans. There are data to suggest that unrepaired meniscus HCTs may develop increased extrusion with time, highlighting the importance of early repair of these types of tears. When meniscus HCTs repaired with all-inside circumferential compression sutures reach a healed state between surgery and 6 months postoperatively and what happens to the repairs at long-term follow-up remains an interesting question and an important area of future research.

\section{Limitations}

This study is not without limitations. First, the study is underpowered to detect a clinically meaningful and significant difference given its limited sample size and is subject to type II error. An observed power of $10 \%$ was calculated using methods described by Hoenig and Heisey. ${ }^{36}$ Only 8 patients were included in this study and meaningful clinical conclusions and practice changing recommendations likely require a larger sample size. Furthermore, given only one surgeon performed all meniscus repairs, the generalizability of results to a broader community of orthopaedic surgeons remains limited.
Although the clinical follow-up data available are relatively short term compared with other published data, this was not the primary end point of this study; rather, MRI and second-look arthroscopic evaluation of healing were the primary endpoints. MRI assessment at 12 months and second-look arthroscopy at 6 months postoperatively are in line with previously published time-points for their evolution respectively. However, it is possible that the promising results seen on IONA (6 months) are due to lower-level activity after repair and the less reassuring results after MRI (12 months) may be due to increased activity. In addition, second-look arthroscopy was evaluated at a single time point.

\section{Conclusions}

Horizontal cleavage meniscal tears repaired with a circumferential compression stitch demonstrate healing on IONA 6 months after surgery. No evidence of incomplete or failed healing was found. MRI at 1 year after surgery demonstrated residual tear evidence for all patients.

\section{References}

1. Englund M, Guermazi A, Gale D, et al. Incidental meniscal findings on knee MRI in middle-aged and elderly persons. N Engl J Med 2008;359:1108-1115.

2. Draijer LW, Belo JN, Berg HF, et al. Summary of the practice guideline 'Traumatic knee problems' (first revision) from the Dutch College of General Practitioners. Ned Tijdschr Geneeskd 2010;154:A2225 [in Dutch].

3. Metcalf MH, Barrett GR. Prospective evaluation of 1485 meniscal tear patterns in patients with stable knees. Am J Sports Med 2004;32:675-680.

4. Bikbaeva AI, Abdurashitov RSh. Combined electrophysiological diagnosis of frontal sinusitis and prognosis of rinosinusogenous intracranial complications. Vestn Otorinolaringol 1991. Mar-Apr:26-30 [in Russian].

5. Woodmass JM, Johnson JD, Wu IT, et al. Horizontal cleavage meniscus tear treated with all-inside circumferential compression stitches. Arthrosc Tech 2017;6:e1329-e1333.

6. Saliman JD. The circumferential compression stitch for meniscus repair. Arthrosc Tech 2013;2:e257-e264. 
7. Furumatsu T, Miyazawa S, Fujii M, et al. Arthroscopic scoring system of meniscal healing following medial meniscus posterior root repair. Int Orthop 2019 05;43: 1239-1245.

8. Lee SS, Ahn JH, Kim JH, et al. Evaluation of healing after medial meniscal root repair using second-look arthroscopy, clinical, and radiological criteria. Am J Sports Med 2018;46:2661-2668.

9. Stelzeneder B, Trabauer BM, Aldrian S, et al. Evaluation of meniscal tissue after meniscal repair using ultrahigh field MRI [published online April, 8, 2020]. J Knee Surg. https://doi.org/10.1055/s-0040-1709135.

10. Willinger L, Herbst E, Diermeier T, et al. High short-term return to sports rate despite an ongoing healing process after acute meniscus repair in young athletes. Knee Surg Sports Traumatol Arthrosc 2019;27:215-222.

11. Fuchs A, Kloos F, Bode G, et al. Isolated revision meniscal repair-failure rates, clinical outcome, and patient satisfaction. BMC Musculoskelet Disord 2018;19:446.

12. Espejo-Reina A, Serrano-Fernández JM, Martín-Castilla B, et al. Outcomes after repair of chronic bucket-handle tears of medial meniscus. Arthroscopy 2014;30:492-496.

13. Tsujii A, Amano H, Tanaka Y, et al. Second look arthroscopic evaluation of repaired radial/oblique tears of the midbody of the lateral meniscus in stable knees. J Orthop Sci 2018;23:122-126.

14. Pujol N, Tardy N, Boisrenoult P, et al. Magnetic resonance imaging is not suitable for interpretation of meniscal status ten years after arthroscopic repair. Int Orthop 2013;37: 2371-2376.

15. Vance K, Meredick R, Schweitzer ME, et al. Magnetic resonance imaging of the postoperative meniscus. Arthroscopy 2009:25:522-530.

16. Zhang K, Crum RJ, Samuelsson K, et al. In-office needle arthroscopy: A systematic review of indications and clinical utility. Arthroscopy 2019;35:2709-2721.

17. McMillan S, Saini S, Alyea E, et al. Office-based needle arthroscopy: A standardized diagnostic approach to the knee. Arthrosc Tech 2017;6:el119-e1124.

18. McIntyre LF. Editorial commentary: What you see is what you get-is in-office needle arthroscopy ready for prime time? Arthroscopy 2019;35:2722-2723.

19. Patel KA, Hartigan DE, Makovicka JL, et al. Diagnostic evaluation of the knee in the office setting using smallbore needle arthroscopy. Arthrosc Tech 2018;7:e17-e21.

20. Quinn R, Lang SD, Gilmer BB. Diagnostic needle arthroscopy and partial medial meniscectomy using small bore needle arthroscopy. Arthrosc Tech 2020;9:e645-e650.

21. Lotysch M, Mink J, Crues JV, et al. Magnetic resonance imaging in the detection of meniscal injuries. Magn Reson Imaging 1986;4:185.

22. Morgan CD, Wojtys EM, Casscells CD, et al. Arthroscopic meniscal repair evaluated by second-look arthroscopy. Am J Sports Med 1991;19:632-637. discussion 7-8.
23. Kanto R, Yamaguchi M, Sasaki K, et al. Second-look arthroscopic evaluations of meniscal repairs associated with anterior cruciate ligament reconstruction. Arthroscopy 2019;35:2868-2877.

24. Goslings JC, Gouma DJ. What is a surgical complication? World J Surg 2008;32:952.

25. Pujol N, Panarella L, Selmi TA, et al. Meniscal healing after meniscal repair: A CT arthrography assessment. Am J Sports Med 2008;36:1489-1495.

26. Hantes ME, Zachos VC, Zibis AH, et al. Evaluation of meniscal repair with serial magnetic resonance imaging: A comparative study between conventional MRI and indirect MR arthrography. Eur J Radiol 2004;50:231-237.

27. Almeida AM, Rodrigues MB, Rezende MU, et al. Meniscal repair results comparing mri, arthro-MRI, and arthro-CT. Acta Ortop Bras 2018;26:166-169.

28. Hoffelner T, Resch H, Forstner R, et al. Arthroscopic allinside meniscal repair-Does the meniscus heal? A clinical and radiological follow-up examination to verify meniscal healing using a 3-T MRI. Skeletal Radiol 201 1;40: $181-187$.

29. Miao Y, Yu JK, Zheng ZZ, et al. MRI signal changes in completely healed meniscus confirmed by second-look arthroscopy after meniscal repair with bioabsorbable arrows. Knee Surg Sports Traumatol Arthrosc 2009;17: 622-630.

30. Ogawa H, Matsumoto K, Sengoku M, et al. Arthroscopic repair of horizontal cleavage meniscus tears provides good clinical outcomes in spite of poor meniscus healing. Knee Surg Sports Traumatol Arthrosc 2020;28:3474-3480.

31. Chen Z, Li WP, Yang R, et al. Meniscal ramp lesion repair using the FasT-Fix technique: Evaluating healing and patient outcomes with second-look arthroscopy. J Knee Surg 2018;31:710-715.

32. Tachibana Y, Sakaguchi K, Goto T, et al. Repair integrity evaluated by second-look arthroscopy after arthroscopic meniscal repair with the FasT-Fix during anterior cruciate ligament reconstruction. Am J Sports Med 2010;38: 965-971.

33. Ahn JH, Kwon OJ, Nam TS. Arthroscopic repair of horizontal meniscal cleavage tears with marrow-stimulating technique. Arthroscopy 2015;31:92-98.

34. Deirmengian CA, Dines JS, Vernace JV, et al. Use of a small-bore needle arthroscope to diagnose intra-articular knee pathology: Comparison with magnetic resonance imaging. Am J Orthop (Belle Mead NJ) 2018;47(2).

35. Amano H, Iwahashi T, Suzuki T, et al. Analysis of displacement and deformation of the medial meniscus with a horizontal tear using a three-dimensional computer model. Knee Surg Sports Traumatol Arthrosc 2015;23: 1153-1160.

36. Hoenig JM, Heisey DM. The abuse of power: The pervasive fallacy of power calculations for data analysis. Am Stat 2001;55:1-6. 